\section{SP0194 PRINCIPLES OF ASSESSMENT IN CLINICAL PRACTICE}

R. Hilfiker. Physiotherapy, HES-SO Valais-Wallis, Leukerbad, Switzerland

In clinical practice, assessment is important for the selection of intervention strategies, goal setting, prognosis and the evaluation of the progression made towards the treatment goals. For example, we need to assess the patients' perceptions on how the disease, functioning and disability interferes with their life situation. Furthermore, we should assess how they can cope with stressful events, what social support they experience and how they adjust to living with a chronic disease. The assessment should provide information on problems, needs and priorities of the patients, as well as on strengths and resources. Based on the initial assessment, mutually agreed treatment goals and treatment strategies can be defined together with the patients.

We need to be aware of the differences, strengths and weaknesses of observations (e.g. Barthel Index), measures (walking speed) and self-reported questionnaires (patient-reported outcomes) or individualised, patient specific measures (e.g. the Patient-Specific Functional Scale).

In most situations, it is recommended to combine generic and specific measures to cover general as well as specific issues of health, functioning and disability or contextual factors. This will also allow the comparison across groups of patients. Each tool used in the assessment should serve a clear objective and should be able to achieve this objective in the given situation. We need to know the relevant measurement properties on reproducibility and validity for the instrument in a given situation. The agreement or the measurement error will tell us, for example, how small a change can be to be detected (i.e. smallest detectable change). This is important when we want to monitor change (evaluation). Reliability indices will tell us how good we can distinguish groups of patients and whether we can use an instrument in a group setting (i.e. in a study; the ICC should be at least 0.7 ) or for the use with individuals in clinical practice (ICC should be at least 0.9).

We can distinguish three types of validity, content validity, construct validity and criterion validity. The content validity informs on how good a tool covers all relevant aspects of the construct to be measured and whether all items cover relevant aspects. An instrument has a good criterion validity if its results are similar to a so-called gold standard (instrument that is known to be valid). The construct validity tells us whether the scores of the measurement instrument are associated with instruments that measure a similar construct and are not associated with scores from instruments that measure non-related concepts.

Other important aspects are responsiveness and interpretability. Responsiveness is the ability of an instrument to detect change over time in the construct to be measured. Interpretability means that the scores are understandable and meaningful. Aspects of interpretability are floor and ceiling effects, and whether the smallest detectable change and the minimal important change are known. Of course, the application of an instrument has to be feasible. Therefore, there is often a trade-off between measurement properties and feasibility, i.e. the perfectly reliable and valid instrument might be too expensive or too time consuming. Therefore, a less "perfect" instrument might be chosen.

All the mentioned aspects need to be considered when defining an individual assessment strategy for a patient. The assessment and evaluation should be an integrated part of the treatment process. It verifies that the treatment is in line with the needs and priorities of the patients; the documentation of changes might motivate both patients and health professionals and it might increase adherence to the recommended treatments.

Disclosure of Interest: None declared

DOI: 10.1136/annrheumdis-2017-eular.7226

\section{SATURDAY, 17 JUNE 2017 \\ HPR Highlight session}

\section{SP0195 HIGHLIGHTS FROM THE HEALTH PROFESSIONAL SESSIONS}

R.J.O. Ferreira ${ }^{1,2} .{ }^{1}$ Health Sciences Research Unit: Nursing (UICiSA:E), Escola Superior de Enfermagem de Coimbra; ${ }^{2}$ Rheumatology department, Centro Hospitalar e Universitário de Coimbra, Coimbra, Portugal

EULAR congress is one on the biggest events in the world of rheumatology. To accommodate all the diversity of topics, several sessions take place at same time, in different rooms. It is very hard, even impossible, to be in all sessions, poster tours, social meetings and other learning events of your interest. It often happens that one want to be at 3 or 4 places at same time.

This "Highlights" session is designed to summarize the most important conclusions of the talks given in all sessions from Health Professionals programme. By this way you can catch on what you missed to be in other places. Of course you can also read the abstracts in the EULAR app or Annals of Rheumatic Diseases Suplement or even to watch the podcasts after the event. However, this highlights session will provide you an integrated view of the different contents in a more appealing and interactive perspective.

Disclosure of Interest: None declared

DOI: 10.1136/annrheumdis-2017-eular.7256 